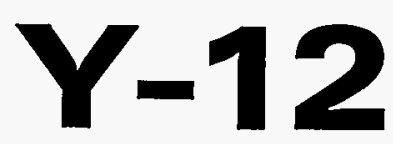

OAK RIDGE $Y-12$ PLANT

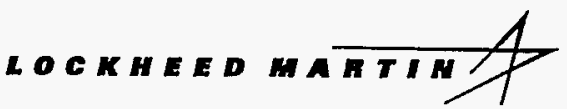

\section{Y-12 PLANT GROUNDWATER PROTECTION PROGRAM GROUNDWATER AND SURFACE WATER SAMPLING AND ANALYSIS PLAN FOR CALENDAR YEAR 1999}

Prepared by

\begin{abstract}
AJA TECHNICAL SERVICES, INC Under Subcontract 70Y-MVM64V
\end{abstract}

September 1998

for the

Environmental Compliance Department Environment, Safety, and Health Organization Oak Ridge Y-12 Plant

Oak Ridge, Tennessee 37831

Managed by

LOCKHEED MARTIN ENERGY SYSTEMS, INC. for the U.S. DEPARTMENT OF ENERGY under contract No. DE-AC05-84OR21400

\section{MANAGED BY}

LOCKHEED MARTIN ENERGY SYSTEMS, INC. FOR THE UNITED STATES

DEPARTMENT OF ENERGY 


\section{DISCLAIMER}

This report was prepared as an account of work sponsored by an agency of the United States Government. Neither the United States Government nor any agency thereof, nor any of their employees, makes any warranty, express or implied, or assumes any legal liability or responsibility for the accuracy, completeness, or usefulness of any information, apparatus, product, or process disclosed, or represents that its use would not infringe privately owned rights. Reference herein to any specific commercial product, process, or service by trade name, trademark, manufacturer, or otherwise, does not necessarily constitute or imply its endorsement, recommendation, or favoring by the United States Government or any agency thereof. The views and opinions of authors expressed herein do not necessarily state or reflect those of the United States Government or any agency thereof. 


\section{DISCLAIMER}

Portions of this document may be illegible electronic image products. Images are produced from the best available original document. 


\section{Y-12 PLANT GROUNDWATER PROTECTION PROGRAM GROUNDWATER AND SURFACE WATER SAMPLING AND ANALYSIS PLAN FOR CALENDAR YEAR 1999}

\section{Prepared by}

AJA TECHNICAL SERVICES, INC Under Subcontract 70Y-MVM64V

September 1998

for the

Environmental Compliance Department Environment, Safety, and Health Organization Oak Ridge Y-12 Plant Oak Ridge, Tennessee 37831

Managed by

LOCKHEED MARTIN ENERGY SYSTEMS, INC. for the U.S. DEPARTMENT OF ENERGY under contract No. DE-AC05-84OR21400 
TABLE OF CONTENTS

Section

Page

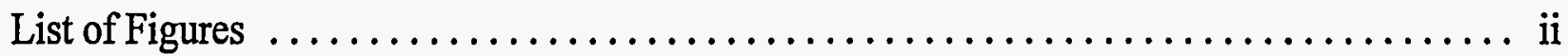

List of Tables ...............................................

List of Acronyms and Abbreviations $\ldots \ldots \ldots \ldots \ldots \ldots \ldots \ldots \ldots \ldots \ldots \ldots \ldots \ldots \ldots \ldots \ldots$ iv

1.0 INTRODUCTION $\ldots \ldots \ldots \ldots \ldots \ldots \ldots \ldots \ldots \ldots \ldots \ldots \ldots \ldots \ldots \ldots \ldots \ldots \ldots .1$

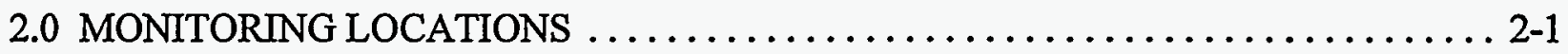

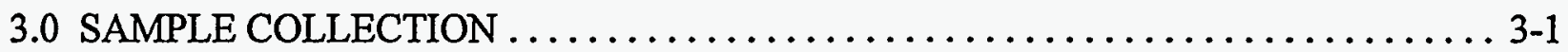

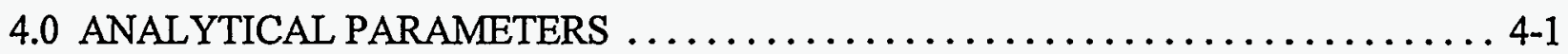

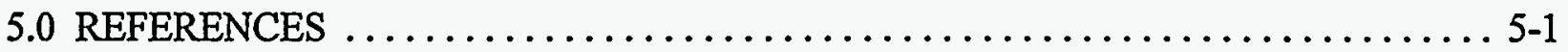

\section{APPENDICES:}
A Figures
B Tables 


\section{List of Figures}

Figure

1 Hydrogeologic Regimes at the Y-12 Plant $\ldots \ldots \ldots \ldots \ldots \ldots \ldots \ldots \ldots \ldots$ A-1

2 Sampling Locations in the Bear Creek Hydrogeologic Regime, CY 1999 . . . . . . A A-2

3 Sampling Locations in the Chestnut Ridge Hydrogeologic Regime, CY 1999 . . . . . A-3

$4 \quad$ Sampling Locations in the Upper East Fork Poplar Creek

Hydrogeologic Regime, CY 1999 .......................... A-4 


\section{List of Tables}

Table

Page

1 Sampling Sequence, Frequency, and Analytical Parameters for Groundwater and Surface Water Monitoring During CY 1999 ....................

2 Field Measurements and Laboratory Analytes for CY 1999 Groundwater and Surface Water Samples . . . . . . . . . . . . . . . . . . . . . . . B-8 


\section{List of Acronyms and Abbreviations}

$\begin{array}{ll}\text { ASO } & \text { Analytical Services Organization } \\ \text { BC } & \text { Bear Creek Hydrogeologic Regime } \\ \text { CR } & \text { Chestnut Ridge Hydrogeologic Regime } \\ \text { CY } & \text { Calendar Year } \\ \text { DOE } & \text { U.S. Department of Energy } \\ \text { EF } & \text { Upper East Fork Poplar Creek Hydrogeologic Regime } \\ \text { GWPP } & \text { Groundwater Protection Program } \\ \text { IWQP } & \text { Integrated Water Quality Program } \\ \text { SESD } & \text { Sampling and Environmental Support Department } \\ \text { SWDF } & \text { Solid Waste Disposal Facility }\end{array}$




\subsection{INTRODUCTION}

This plan provides a description of the groundwater and surface water quality monitoring activities planned for calendar year (CY) 1999 at the U.S. Department of Energy (DOE) Y-12 Plant that will be managed by the Y-12 Plant Environment, Safety, and Health Organization through the Y-12 Plant Groundwater Protection Program (GWPP). Groundwater and surface water monitoring during CY 1999 will be performed in three hydrogeologic regimes at the Y-12 Plant: the Bear Creek Hydrogeologic Regime (Bear Creek Regime), the Upper East Fork Poplar Creek Hydrogeologic Regime (East Fork Regime), and the Chestnut Ridge Hydrogeologic Regime (Chestnut Ridge Regime). The Bear Creek and East Fork regimes are located in Bear Creek Valley, and the Chestnut Ridge Regime is located south of the Y-12 Plant (Figure 1).

Groundwater and surface water monitoring performed under the auspices of the Y-12 Plant GWPP during CY 1999 will comply with:

- Tennessee Department of Environment and Conservation regulations governing detection monitoring at nonhazardous Solid Waste Disposal Facilities (SWDF); and

- DOE Order 5400.1 surveillance monitoring and exit pathway/perimeter monitoring.

Some of the data collected for these monitoring drivers will also be used to meet monitoring requirements of the Integrated Water Quality Program (IWQP), which is managed by Bechtel Jacobs Company LLC. Data from five sampling locations collected for SWDF monitoring in the Chestnut Ridge Regime will be used to comply with requirements specified in Resource Conservation and Recovery Act post-closure permit regarding corrective action monitoring. Also, data from sampling locations in each regime will be provided to the IWQP as requested.

Modifications to the CY 1999 monitoring program may be necessary during implementation. Changes in regulatory or programmatic requirements may alter the analytes specified for selected monitoring wells, or wells could be added or removed from the planned monitoring network. All modifications to the monitoring program will be approved by the Y-12 Plant GWPP manager and documented as addenda to this sampling and analysis plan. 


\subsection{MONITORING LOCATIONS}

Groundwater samples will be collected from a total of 80 monitoring wells, including 32 wells located in the Bear Creek Regime (Figure 2), 17 wells located in the Chestnut Ridge Regime (Figure 3), and 31 wells located in the East Fork Regime (Figure 4). Samples of groundwater discharging from 10 natural springs also will be collected, including four springs (SS-1, SS-4, SS-5, and SS-6) in the Bear Creek Regime (Figure 2) and six springs (SCR2.1SP, SCR2.2SP, SCR3.4SP, SCR4.3SP, SCR5.1SP, and SCR5.4SP) in the Chestnut Ridge Regime (Figure 3).

Surface water samples will be collected from a total of 12 sampling locations during CY 1999, including 11 locations in the Bear Creek Regime and one location in the East Fork Regime. In the Bear Creek Regime, samples will be collected from Bear Creek at six sampling stations located from about 0.6 to 12 kilometers upstream of the confluence of Bear Creek and East Fork Poplar Creek (BCK-00.63 to BCK-11.97), and from five sampling stations along northern tributaries to Bear Creek (NT-01, NT-02, NT-06, NT-07, and NT-08) (Figure 2). In the East Fork Regime, samples will be collected from the Lake Reality Emergency Spillway (LRSPW) (Figure 4). 


\subsection{SAMPLE COLLECTION}

Groundwater and surface water samples will be collected by personnel from the Sampling and Environmental Support Department (SESD) of the Analytical Services Organization (ASO). Groundwater sampling will be performed in accordance with the most recent version of the technical procedure approved by the Y-12 Plant GWPP Manager: Groundwater Sampling (Lockheed Martin Energy Systems, Inc. 1997). Procedures for obtaining field measurements and for surface water sampling are contained in Environmental Surveillance Procedures Quality Control Manual (Lockheed Martin Energy Systems, Inc. 1988). Sample collection, handling, analysis, and surveillance will be performed in accordance with the GWPP Quality Program Plan (Science Applications International Corporation 1994), and the ASO Laboratory Quality Assurance Project Plan for groundwater monitoring at the Y-12 Plant (Martin Marietta Energy Systems 1991).

Monitoring wells, springs, and surface water stations are subdivided into sample groups based on hydrogeologic regimes. These sample groups, located in the Bear Creek (BC), Chestnut Ridge (CR), and East Fork (EF) hydrogeologic regimes, will be sampled in the sequence shown on Table 1. The sampling sequence is generally from least contaminated wells to most contaminated wells at each location within each sampling group. Groundwater samples will be collected using dedicated sampling equipment (Well Wizard ${ }^{\mathrm{TM}}$ bladder pumps) unless a well is scheduled for onetime sampling. For monitoring wells that are nested closely together or that are equipped with a Westbay ${ }^{\mathrm{TM}}$ multiport sampling system, the collection sequence is from shallowest to deepest monitored interval. For surface water and springs along Bear Creek, sampling is from the furthest downstream locations to the upstream location closest to the S-3 Site (Table 1).

Samples will be collected semiannually from all of the monitoring wells, springs, and surface water stations in each regime, with the exception of sampling groups BC-4(eight wells and four surface water stations), BC-WB (eight sampling ports of Westbay ${ }^{\mathrm{TM}}$ well GW-726), and EF-4 (two wells) that are scheduled for one-time sampling during CY 1999. As summarized below, the number of samples to be collected during each quarter will range from 36 to 80 , for an annual total of 214 samples.

\begin{tabular}{|r|c|c|c|c|c|}
\hline HYDROGEOLOGIC & \multicolumn{3}{|c|}{ NUMBER OF SAMPLES PER QUARTER OF CY 1999 } \\
\cline { 2 - 6 } & REGIME & 1st & 2nd & 3rd & 4th \\
\hline BC Regime & 54 & 0 & 34 & 0 \\
CR Regime & 16 & 7 & 16 & 7 \\
EF Regime & 10 & 29 & 10 & 31 \\
\hline TOTAL: & $\mathbf{8 0}$ & 36 & 60 & 38 \\
\hline
\end{tabular}


Groundwater samples from all monitoring wells, except those equipped with a Westbay ${ }^{\mathrm{TM}}$ multiport sampling system, will be collected using the low-flow minimal drawdown purging and sampling method. Sample collection using this method began in October 1997 and initial results show a significant reduction in sample turbidity and increased similarity between total (unfiltered) and dissolved (filtered) metal concentrations. Beginning in CY 1999, filtered samples will be collected only from specified wells (Table 1), including all of the sample groups in the Chestnut Ridge Regime and sample groups BC-4, BC-WB, EF-3, and EF-WB.

In addition to the groundwater and surface water samples, field blanks and equipment rinsate samples will be collected at the frequencies and analyzed for the parameters specified on Table 1. Field blank samples will be collected from at least $10 \%$ of the sample groups. Equipment rinsate samples will be collected from wells in sample groups with nondedicated sampling equipment (BC-4) or with Westbay ${ }^{\mathrm{TM}}$ multiport sampling systems (BC-WB and EF-WB). The rinsate sample will be collected at the last sampling port (Westbay ${ }^{\mathrm{TM}}$ ) or the last well sampled with each pump immediately following the field cleaning procedure.

Trip blank samples, field duplicate samples, and laboratory quality assurance samples will be prepared and analyzed as specified in the ASO Laboratory Quality Assurance Project Plan (Martin Marietta Energy Systems, Inc. 1991) using applicable analytical procedures. The location (building and room number) where the trip blank samples are prepared will be recorded on the field data sheets. Trip blank samples will be prepared for each cooler used to transport samples for volatile organic analyses. Duplicate samples will be collected from at least $10 \%$ of the sampling locations. A total of 26 field duplicate samples will be collected during CY 1999, including eight in the Bear Creek Regime, ten in the Chestnut Ridge Regime, and eight in the East Fork Regime (Table 1). 


\subsection{ANALYTICAL PARAMETERS}

The monitoring drivers for CY 1999 require an extensive list of analytes (Table 2). For this Sampling and Analysis Plan, specific analytes are grouped by analytical method or by type (e.g., metals) and referenced as analytical parameters. All groundwater and surface water samples will be analyzed for the following suite of parameters (identified as the Standard Administrative Parameter Group):

- field measurements - water level (monitoring wells), temperature, $\mathrm{pH}$, conductivity, dissolved oxygen, and oxidation-reduction potential;

- $\quad$ miscellaneous laboratory analytes - $\mathrm{pH}$, conductivity, turbidity, total suspended solids, and total dissolved solids;

- major anions;

- $\quad$ trace metals (includes major cations);

- a comprehensive suite of organic compounds; and

- gross alpha and gross beta activity.

The Y-12 Plant National Pollution Elimination System Program requested results for several radionuclides in addition to the analytes included in the Standard Administrative Parameter Group for surface water samples from BCK-11.97 in BC-3 (Table 1). Additional radionuclides also will be reported for groundwater samples from the wells scheduled for one-time sampling in EF-4 (Table 1). 


\subsection{REFERENCES}

Martin Marietta Energy Systems, Inc. 1991. Laboratory Quality Assurance Project Plan for the Sampling and Analysis of Groundwater Wells at the Y-12 Plant Site on the Oak Ridge Reservation. Prepared by Analytical Chemistry Department Technical Division, QAP: 04-90-0014.

Lockheed Martin Energy Systems, Inc. 1988. Environmental Surveillance Procedures Quality Control Manual. (ES/ESH/MNT-14).

Lockheed Martin Energy Systems, Inc. 1997. Groundwater Sampling. Prepared by the Analytical Services Organization (SESD-TP-8204, Rev. 3).

Science Applications International Corporation. 1994. Groundwater Protection Program Quality Program Plan. Prepared for Martin Marietta Energy Systems, Inc. (Y/SUB/94-99069C/Y13/1).

U.S. Environmental Protection Agency. 1983. Methods for Chemical Analysis of Water and Wastes.

U.S. Environmental Protection Agency. 1986. Test Methods for Evaluating Solid Waste Physical/Chemical Methods. 


\section{APPENDIX A}

Figures 


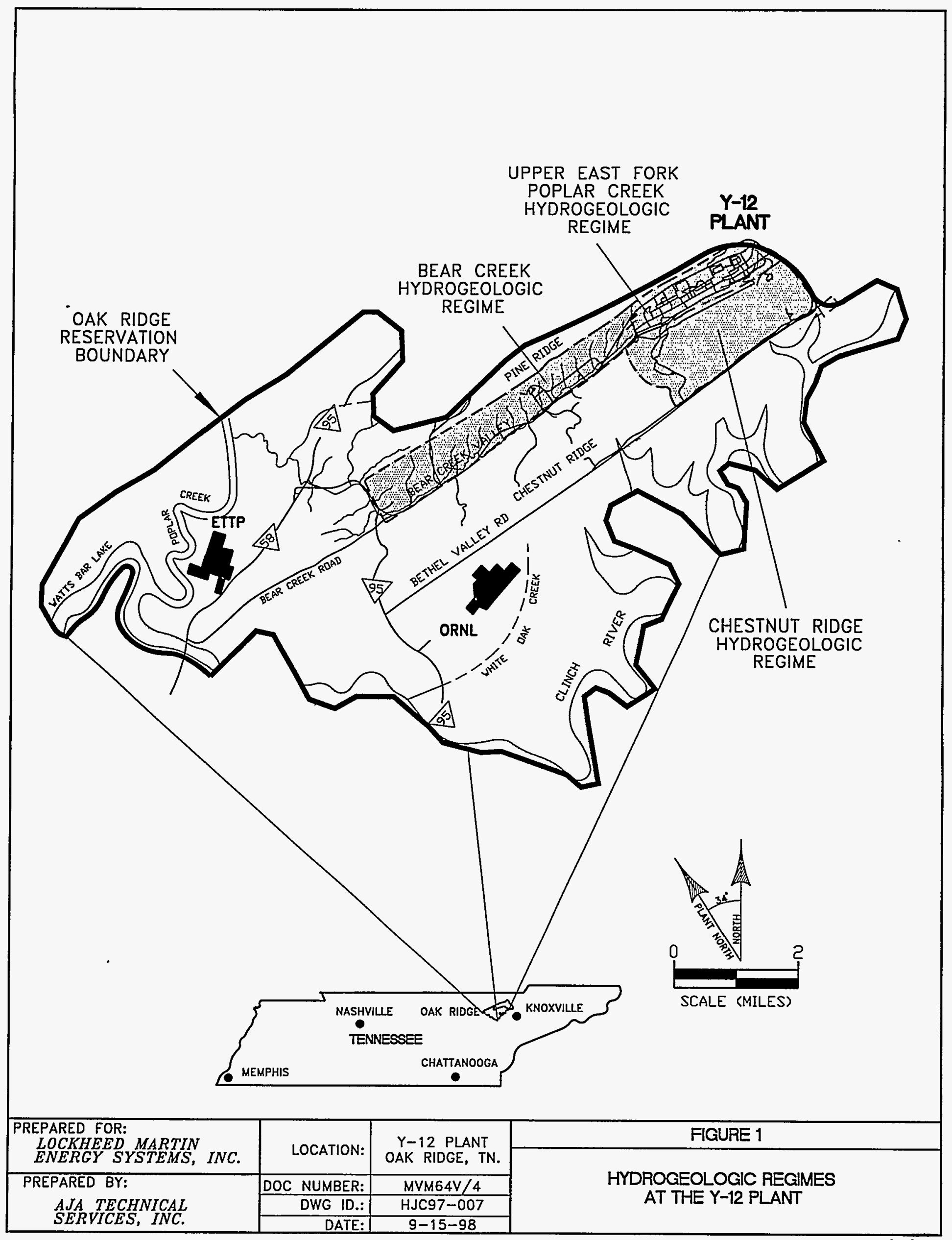



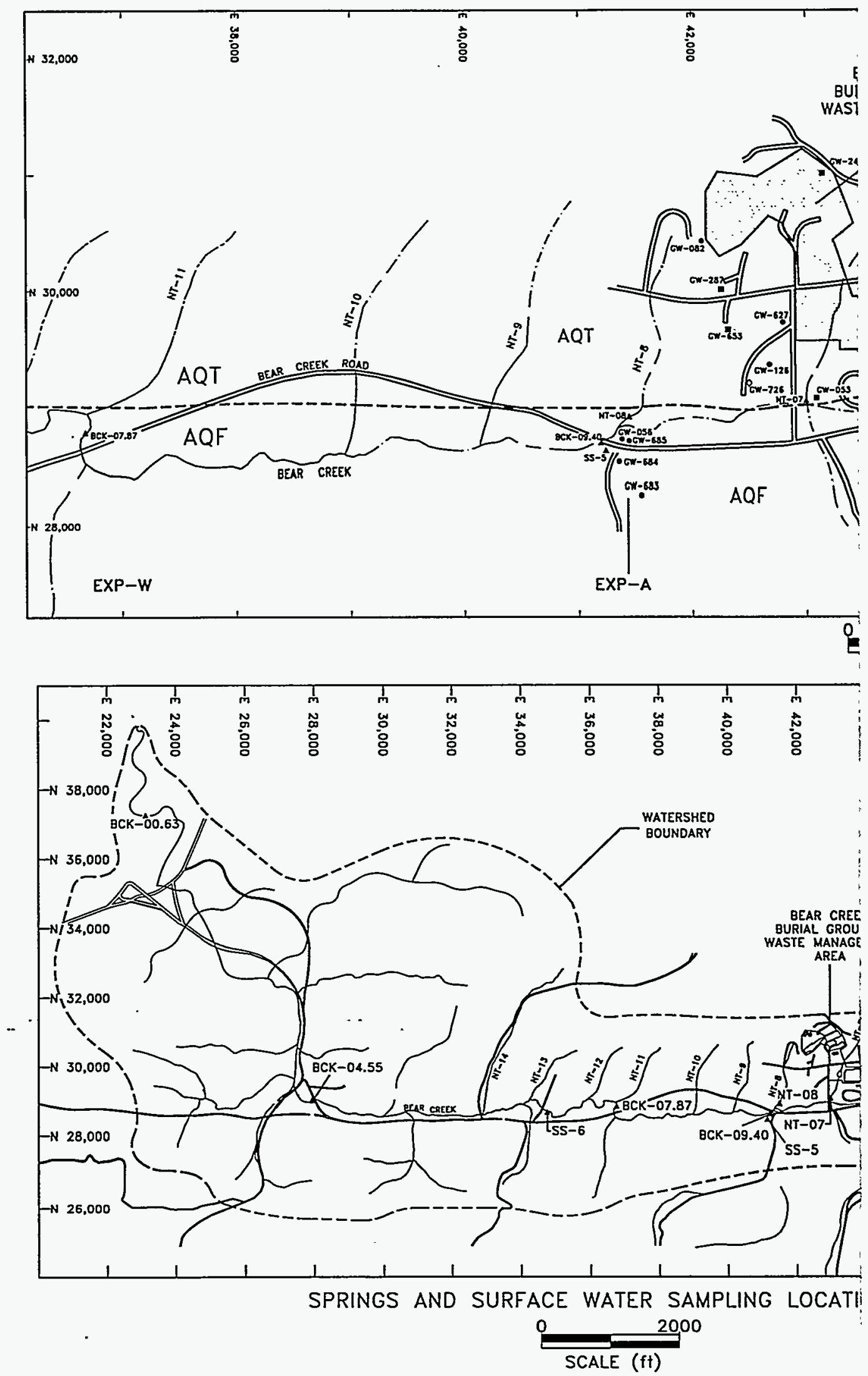


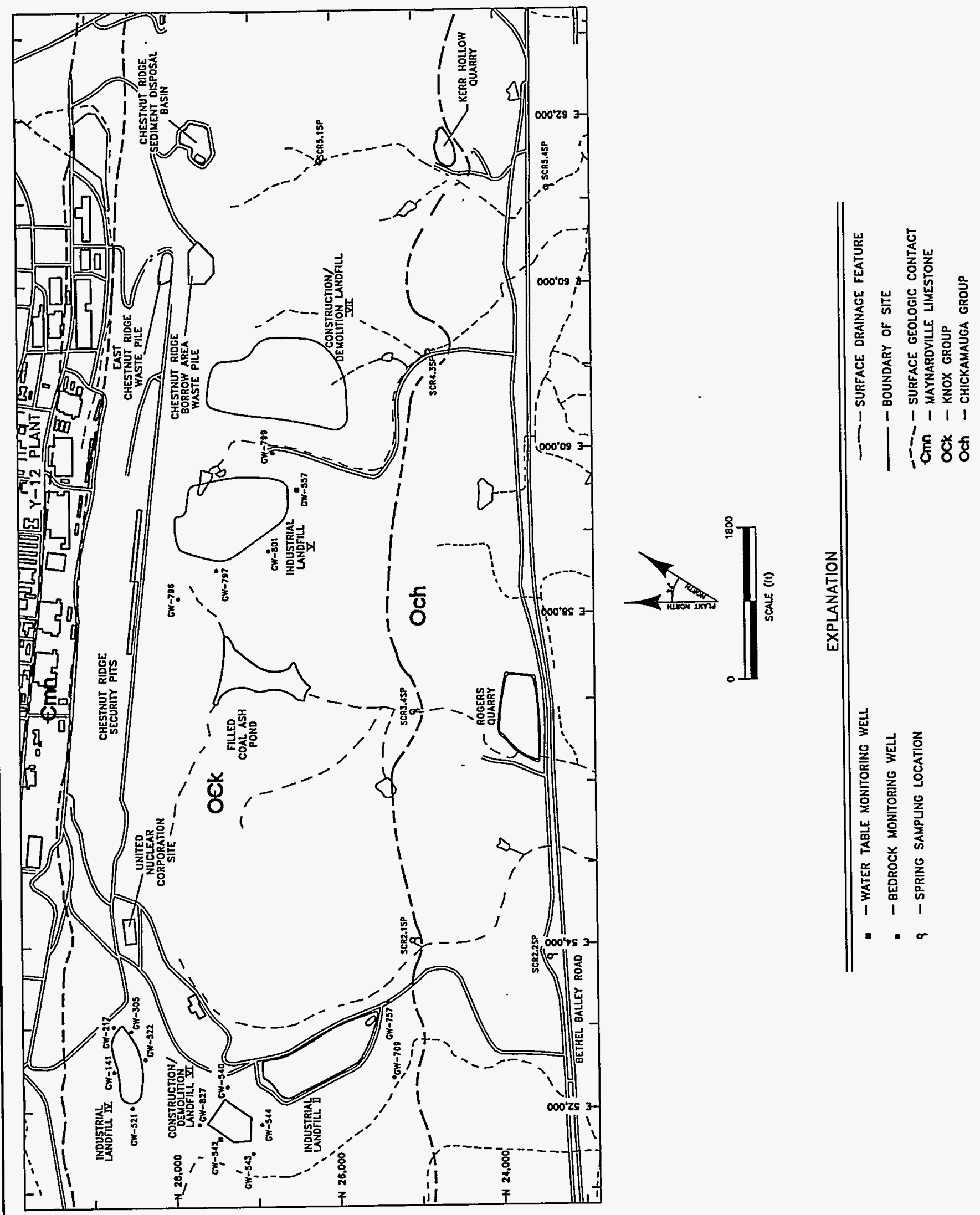

\begin{tabular}{|c|r|c|}
\hline $\begin{array}{c}\text { PREPARED FOR: } \\
\text { LOCKHEED MARTIN } \\
\text { ENERGY SYSTEMS, INC. }\end{array}$ & LOCATION: & $\begin{array}{c}\text { Y-12 PLANT } \\
\text { OAK RIDGE, TN. }\end{array}$ \\
\hline PREPARED BY: & DOC NUMBER: & MVM64V/4 \\
\cline { 2 - 3 } AJA TECHNICAL & DWG ID.: & $\mathrm{HJC} 96-033$ \\
\cline { 2 - 2 } SERVICES, INC. & DATE: & $9-15-98$ \\
\hline
\end{tabular}

FIGURE 3

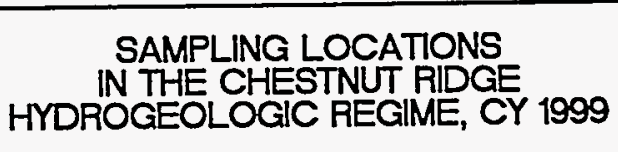




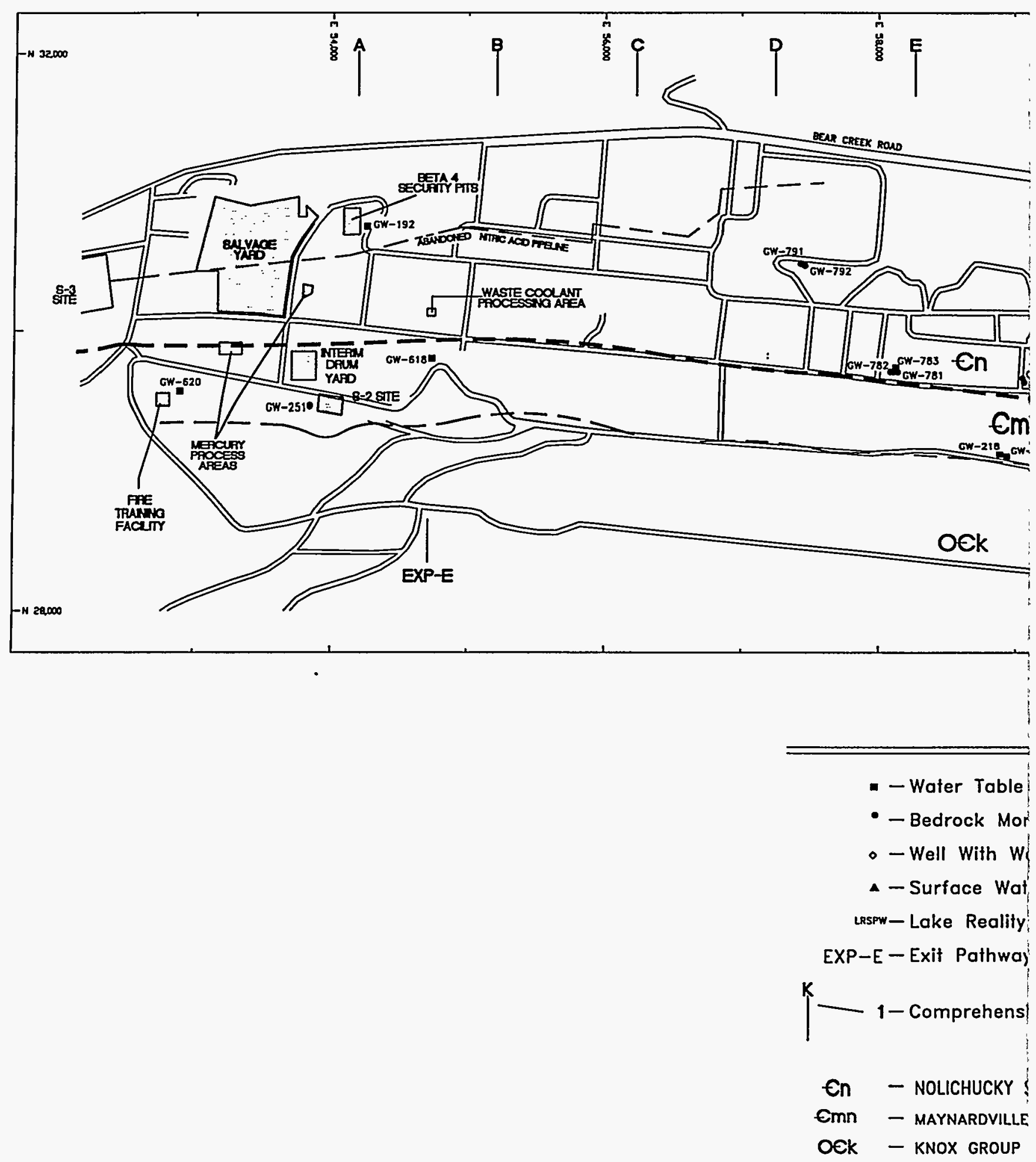


APPENDIX B

Tables 


\section{Table 1.}

Sampling Sequence, Frequency, and Analytical Parameters for

Groundwater and Surface Water Monitoring During CY 1999

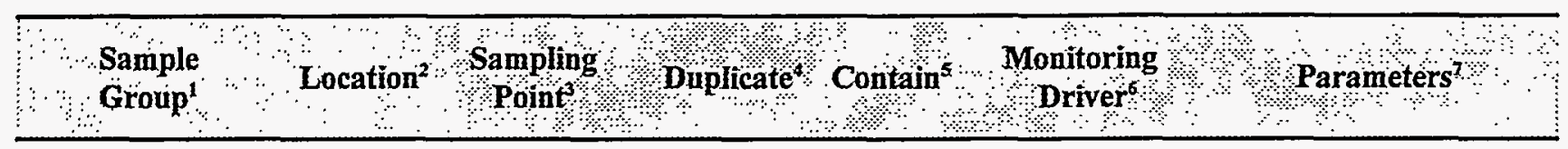

Bear Creek Hydrogeologic Regime

\begin{tabular}{|c|c|c|c|c|c|c|}
\hline BC-1 & EXP-A & GW-056 & & & EXP & STD \\
\hline \multirow{12}{*}{$(\mathrm{Q} 1, \mathrm{Q} 3)$} & EXP-A & GW-685 & & & EXP & STD \\
\hline & EXP-A & GW-684 & & $Y$ & EXP & STD \\
\hline & EXP-A & GW-683 & & $\mathrm{Y}$ & EXP & STD \\
\hline & EXP-B & GW-621 & & & EXP & STD \\
\hline & EXP-B & GW-695 & - & & EXP & STD \\
\hline & EXP-B & GW-703 & & $\mathrm{Y}$ & EXP & STD \\
\hline & EXP-B & GW-704 & & $Y$ & EXP & STD \\
\hline & EXP-B & GW-706 & Q1 & $\mathrm{Y}$ & EXP & STD \\
\hline & EXP-C & GW-740 & & $\mathrm{Y}$ & EXP & STD \\
\hline & EXP-C & GW-738 & & $\mathrm{Y}$ & EXP & STD \\
\hline & EXP-C & GW-724 & & $\mathrm{Y}$ & EXP & STD \\
\hline & EXP-C & GW-725 & Q3 & $Y$ & EXP & STD \\
\hline BC-2 & BG & GW-287 & & & SMP & STD \\
\hline \multirow[t]{9}{*}{ (Q1, Q3) } & BG & GW-653 & & $\mathrm{Y}$ & SMP & STD \\
\hline & BG & GW-053 & & $\mathrm{Y}$ & SMP & STD \\
\hline & BG & GW-627 & Q1 & $\mathrm{Y}$ & SMP & STD \\
\hline & SPI & GW-315 & & $\mathrm{Y}$ & SMP & STD \\
\hline & $\mathrm{RS}$ & GW-311 & & $\mathrm{Y}$ & SMP & STD \\
\hline & OLF & GW-829 & & $\mathrm{Y}$ & SMP & STD \\
\hline & OLF & GW-085 & & $\mathrm{Y}$ & SMP & STD \\
\hline & OLF & GW-537 & & $\mathrm{Y}$ & SMP & STD \\
\hline & OLF & GW-226 & Q3 & $Y$ & SMP & STD \\
\hline
\end{tabular}


Table 1 (cont'd)

\begin{tabular}{|c|c|c|c|c|c|c|}
\hline $\begin{array}{l}\text { Sample } \\
\text { Group }\end{array}$ & Location ${ }^{2}$ & Sampling & puplicaté & Contains & $\begin{array}{l}\text { Monitoring } \\
\text { Driver }\end{array}$ & 3 Parameters \\
\hline $\mathrm{BC}-3$ & EXP-SW & BCK-00.63 & & & EXP & STD \\
\hline \multirow[t]{2}{*}{ (Q1, Q3) } & EXP-SW & BCK-04.55 & & & EXP & STD \\
\hline & EXP-SW. & SS-6 & & & EXP & STD \\
\hline \multirow[t]{8}{*}{ - } & EXP-SW & BCK-07.87 & Q1 & & EXP & STD \\
\hline & EXP-SW & SS-5 & & & $\operatorname{EXP}$ & STD \\
\hline & EXP-SW & BCK-09.40 & & & EXP & STD \\
\hline & EXP-SW & SS-4 & Q3 & & EXP & STD \\
\hline & EXP-SW & BCK-10.60 & & & EXP & STD \\
\hline & EXP-SW & BCK-11.97 & & & EXP & $\operatorname{STD}, \operatorname{RAD}(2,3,4,5,8,13)$ \\
\hline & EXP-SW & SS-1 & & & EXP & STD \\
\hline & EXP-SW & NT-01 & & & EXP & STD \\
\hline $\mathrm{BC}-4$ & EXP-SW & NT-08 & & & SMP & STD \\
\hline \multirow[t]{12}{*}{ (Q1) } & EXP-SW & NT-07 & & & SMP & STD \\
\hline & EXP-SW & NT-06 & & & SMP & STD \\
\hline & EXP-SW & NT-02 & & & SMP & STD \\
\hline & BG & GW-126 & & $\mathrm{Y}$ & SMP & STD/F \\
\hline & BG & GW-082 & & $Y$ & SMP & STD/F \\
\hline & $\mathrm{BG}$ & GW-242 & & $\mathrm{Y}$ & SMP & $\mathrm{STD} / \mathrm{F}$ \\
\hline & OLF & GW-601 & & $\mathrm{Y}$ & SMP & $\mathrm{STD} / \mathrm{F}$ \\
\hline & S3 & GW-345 & & $\mathrm{Y}$ & SMP & STD/F \\
\hline & S3 & GW-346 & & $\mathrm{Y}$ & SMP & $\mathrm{STD} / \mathrm{F}$ \\
\hline & \$3 & GW-526 & Q1 & $\mathrm{Y}$ & SMP & $\mathrm{STD} / \mathrm{F}$ \\
\hline & OLF & GW-087 & & $\mathrm{Y}$ & SMP & STD/F \\
\hline & \multicolumn{3}{|c|}{ RINSATE SAMPLE } & & & STD \\
\hline BC-WB & $\mathrm{BG}$ & GW-726-23 & & & SMP & STD/F \\
\hline \multirow[t]{3}{*}{ (Q1) } & $B G$ & GW-726-20 & & & SMP & $\mathrm{STD} / \mathrm{F}$ \\
\hline & BG & GW-726-16 & & & SMP & STD/F \\
\hline & BG & GW-726-12 & & & SMP & STD/F \\
\hline
\end{tabular}

B-2 
Table 1 (cont'd)

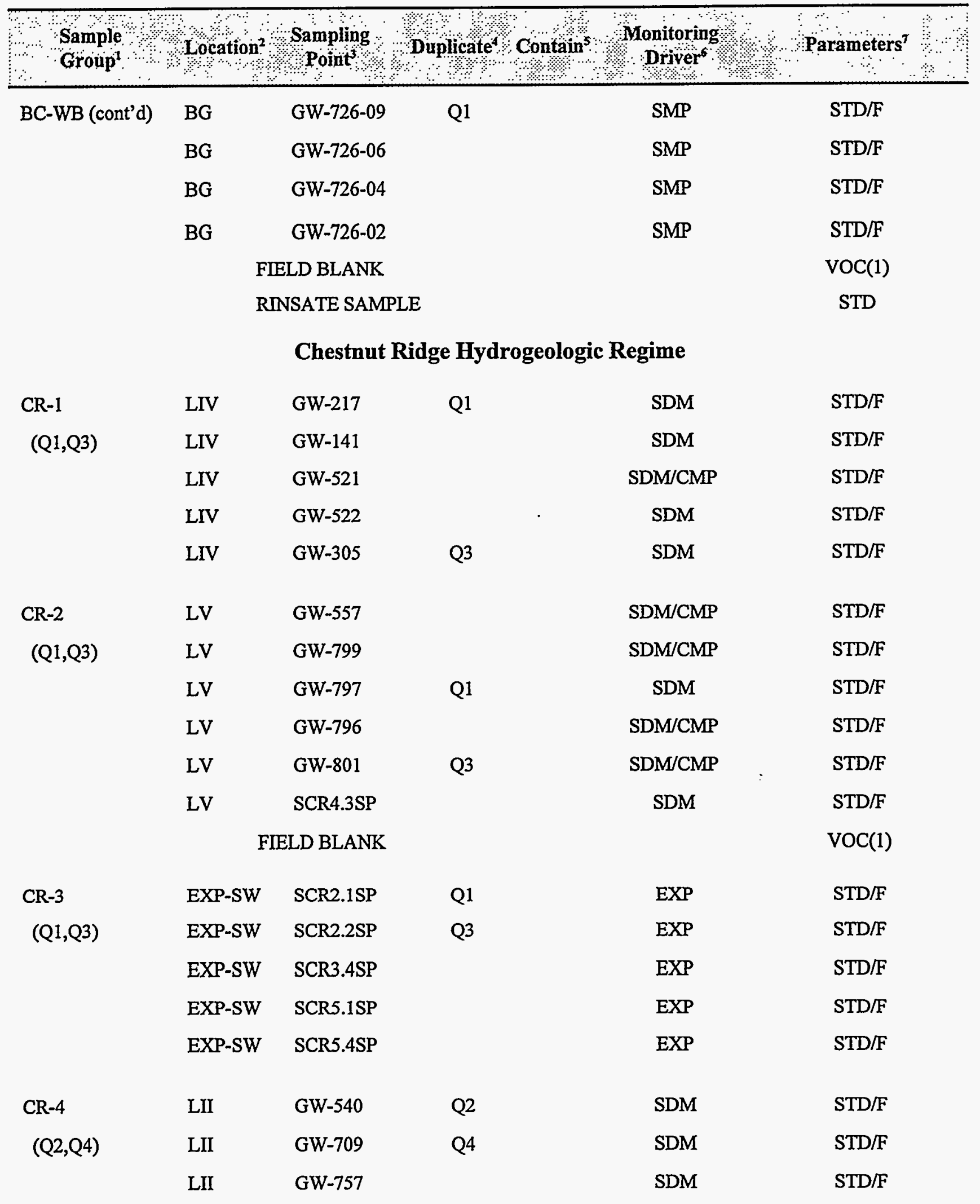


Table 1 (cont'd)

\begin{tabular}{|c|c|c|c|c|c|c|}
\hline $\begin{array}{c}\text { Sample } \\
\text { Group? }\end{array}$ & Location $^{2}$ & $\begin{array}{l}\text { Samping } \\
\text { Point } \\
\end{array}$ & Duplicaté & $\begin{array}{l}\text { Contain's } \\
\text { Pे }\end{array}$ & $\begin{array}{l}\text { Monitoring } \\
\text { Driver"? }\end{array}$ & Parameters \\
\hline CR-5 & CDLVI & GW-827 & Q2 & & SDM & STD/F \\
\hline \multirow[t]{3}{*}{ (Q2, Q4) } & CDLVI & GW-542 & Q4 & & SDM & STD/F \\
\hline & CDLVI & GW-543 & & & SDM & STD/F \\
\hline & CDLVI & GW-544 & & & SDM & $\mathrm{STD} / \mathrm{F}$ \\
\hline \multicolumn{7}{|c|}{ Upper East Fork Poplar Creek Hydrogeologic Regime } \\
\hline EF-1 & GRID F3 & GW-789 & & & SMP & STD \\
\hline \multirow[t]{10}{*}{$(\mathrm{Q} 2, \mathrm{Q})$} & GRID F3 & GW-788 & & & SMP & STD \\
\hline & GRID G3 & GW-770 & & & SMP & STD \\
\hline & GRID G3 & GW-769 & & $\mathrm{Y}$ & SMP & STD \\
\hline & GRID H3 & GW-776 & & & SMP & STD \\
\hline & GRID H3 & GW-775 & & $\mathrm{Y}$ & SMP & STD \\
\hline & GRID E3 & GW-783 & & $\mathrm{Y}$ & SMP & STD \\
\hline & GRID E3 & GW-782 & Q2 & $\mathrm{Y}$ & SMP & STD \\
\hline & GRID E3 & GW-781 & & $\mathrm{Y}$ & SMP & STD \\
\hline & GRID D2 & GW-792 & & $\mathrm{Y}$ & SMP & STD \\
\hline & GRID D2 & GW-791 & Q4 & $\mathrm{Y}$ & SMP & STD \\
\hline EF-2 & B4 & GW-192 & & $\mathrm{Y}$ & SMP & STD \\
\hline \multirow[t]{9}{*}{$(\mathrm{Q} 2, \mathrm{Q} 4)$} & EXP-E & GW-618 & & $\mathrm{Y}$ & EXP & STD \\
\hline & S2 & GW-251 & Q2 & $\mathrm{Y}$ & EXP & STD \\
\hline & FTF & GW-620 & & $\mathrm{Y}$ & EXP & STD \\
\hline & GRID JP & GW-763 & & $\mathrm{Y}$ & SMP & STD \\
\hline & NHP & GW-148 & & $\mathrm{Y}$ & SMP & STD \\
\hline & NHP & GW-153 & Q4 & $\mathrm{Y}$ & EXP & STD \\
\hline & NHP & GW-380 & & $\mathrm{Y}$ & EXP & STD \\
\hline & NHP & GW-220 & & $\mathrm{Y}$ & EXP & STD \\
\hline & NHP & GW-383 & & $\mathrm{Y}$ & SMP & STD \\
\hline EF-3 & EXP-SW & LRSPW & & & EXP & $\mathrm{STD} / \mathrm{F}$ \\
\hline \multirow[t]{3}{*}{$(\mathrm{Q} 2, \mathrm{Q} 4)$} & EXP-SR & GW-207 & & & EXP & STD/F \\
\hline & EXP-SR & GW-208 & & & EXP & $\mathrm{STD} / \mathrm{F}$ \\
\hline & EXP-SR & GW-816 & Q2 & & EXP & STD/F \\
\hline
\end{tabular}




\section{Table 1 (cont'd)}

\begin{tabular}{|c|c|c|c|c|c|c|}
\hline $\begin{array}{l}\text { Sample } \\
\text { Group }\end{array}$ & Location & Sampling & Duplicate & Contain & Monitoring & Parameters \\
\hline \multirow[t]{4}{*}{ EF-3 (cont'd) } & GRID K1 & GW-744 & & & SMP & STD/F \\
\hline & GRID K2 & GW-747 & & & SMP & STD/F \\
\hline & EXP-J & GW-735 & & & EXP & STD/F \\
\hline & EXP-J & GW-750 & Q4 & & EXP & $\mathrm{STD} / \mathrm{F}$ \\
\hline $\mathrm{EF}-4$ & UOV & GW-218 & & $\mathrm{Y}$ & SMP & STD/F, RAD $(2,3,4,5,8)$ \\
\hline (Q4) & UOV & GW-219 & Q4 & $\mathrm{Y}$ & SMP & $\mathrm{STD} / \mathrm{F}, \mathrm{RAD}(2,3,4,5,8)$ \\
\hline EF-WB & EXP-J & GW-722-33 & & & EXP & STD/F \\
\hline \multirow[t]{11}{*}{$(\mathrm{Q} 1, \mathrm{Q} 3)$} & EXP-J & GW-722-32 & & & EXP & STD/F \\
\hline & EXP-J & GW-722-30 & & & EXP & STD/F \\
\hline & EXP-J & GW-722-26 & & & EXP & $\mathrm{STD} / \mathrm{F}$ \\
\hline & EXP-J & GW-722-22 & Q1 & & EXP & STD/F \\
\hline & EXP-J & GW-722-20 & Q3 & & EXP & STD/F \\
\hline & EXP-J & GW-722-17 & & & EXP & STD/F \\
\hline & EXP-J & GW-722-14 & & & EXP & $\mathrm{STD} / \mathrm{F}$ \\
\hline & EXP-J & GW-722-10 & & & EXP & STD/F \\
\hline & EXP-J & GW-722-06 & & & EXP & $\mathrm{STD} / \mathrm{F}$ \\
\hline & \multicolumn{3}{|c|}{ FIELD BLANK } & & & VOC(1) \\
\hline & \multicolumn{3}{|c|}{ RINSATE SAMPLE } & & & STD \\
\hline
\end{tabular}

Notes:

1 Samples will be collected during the calendar year quarter as specified (e.g., Q1). Surface water and spring samples in BC-3 will be collected on or about the same day as groundwater samples will be collected from wells GW-683 and GW-684 in BC-1.

Bear Creek Regime

BG - Bear Creek Burial Grounds Waste Management Area

EXP - Exit Pathway Monitoring Location: Maynardville Limestone Picket (-A, $-\mathrm{B},-\mathrm{C})$

Spring or Surface Water Location (-SW)

OLF - Oil Landfarm Waste Management Area

RS - Rust Spoil Area

S3 - S-3 Site

SPI - Spoil Area I 


\section{Table 1 (cont'd)}

\section{Notes (cont'd):}

\section{Chestnut Ridge Regime}

CDLVI - Construction/Demolition Landfill VI

EXP-SW - Exit Pathway Monitoring Location (spring)

LII - Industrial Landfill II

LIV - Industrial Landfill IV

LV - Industrial Landfill V

\section{East Fork Regime}

B4 - Beta-4 Security Pits

EXP - Exit Pathway Monitoring Location: Maynardville Limestone Picket (-E, -J)

Along Scarboro Road in the gap through Pine Ridge (-SR)

FTF - Fire Training Facility

GRID - Comprehensive Groundwater Monitoring Plan Grid Location

NHP - New Hope Pond

UOV - Uranium Oxide Vault

S2 - S-2 Site

3 BCK - Bear Creek Kilometer (Surface Water Sampling Station)

GW - Groundwater Monitoring Well

LRSPW - Lake Reality Spillway (Surface Water Sampling Station)

NT - North Tributary to Bear Creek

SCR - South Chestnut Ridge (Spring Sampling Station)

SS - Spring Sampling Location: South Side of Bear Creek

4 Q - Field duplicate samples will be collected at these locations during the quarter specified.

$5 \quad \mathrm{Y}-$ All purged groundwater will be contained at these locations.

6 CMP - RCRA Post-Closure Corrective Action Monitoring

EXP - DOE Order 5400.1 Exit Pathway Monitoring

SDM - SWDF Detection Monitoring

SMP - DOE Order 5400.1 Surveillance Monitoring

7 Table 2 provides a comprehensive list of analytes and analytical methods grouped by parameter.

/F - Filtered sample for dissolved metal concentrations.

STD - Standard administrative parameter group.

See the following list of parameters that apply to CY 1999 samples. 


\section{Table 1 (cont'd)}

\section{Notes (cont'd):}

Standard Administrative Parameter Group:

FLD - Field measurements

CHEM - Miscellaneous laboratory analytes (e.g., $\mathrm{pH}$ ) and anions

MET(1) - Metals; analyzed by inductively coupled plasma spectrometry, except those analyzed by:

- plasma mass spectrometry antimony, arsenic, cadmium, lead, thallium, and uranium

- cold vapor atomic absorption

$$
\text { mercury }
$$

VOC(1) - Volatile organic compounds

$\mathrm{RAD}(1)$ - Gross alpha and gross beta

\section{Additional Radionuclides:}

$\operatorname{RAD}(2)$ - Strontium-89/90, technetium-99, and tritium

$\mathrm{RAD}(3)$ - Uranium-234, -235 , and -238

$\mathrm{RAD}(4)$ - Americium-241, iodine-129, neptunium-237, plutonium-238 and -239/240

$\mathrm{RAD}(5)$ - Radium-223/224/226

$\operatorname{RAD}(8)$ - Thorium-228, $-230,-232$, and -234

$\mathrm{RAD}(13)$ - Total uranium and weight percent U-235 
Table 2.

Field Measurements and Laboratory Analytes for CY 1999 Groundwater and Surface Water Samples

\begin{tabular}{|c|c|c|c|}
\hline FLD _ Field Measurements & Analytical Method? & Detection Limit. & Units $^{3}$ \\
\hline Depth to Water & ESP 302-1 & NA & $\mathrm{ft}$ \\
\hline Water Temperature & ESP 307-1 & NA & centigrade \\
\hline $\mathrm{pH}$ & ESP 307-1 & NA & $\mathrm{pH}$ units \\
\hline Conductivity & ESP 307-1 & $\mathrm{NA}$ & $\mu \mathrm{mho} / \mathrm{cm}$ \\
\hline Dissolved Oxygen & ESP $307-3$ & NA & $\mathrm{ppm}$ \\
\hline Oxidation-Reduction Potential & ESP $307-5$ & NA & $\mathrm{mV}$ \\
\hline CHEM - Miscellaneous Laboratory Analytes & अ. & \% & \% \\
\hline $\mathrm{pH}$ & EPA-9040 & NA & pH units \\
\hline Conductivity & EPA-9050 & $\mathrm{NA}$ & $\mu \mathrm{mho} / \mathrm{cm}$ \\
\hline Total Dissolved Solids & EPA-160.1 & 1 & $\mathrm{mg} / \mathrm{L}$ \\
\hline Total Suspended Solids & EPA-160.2 & 1 & $\mathrm{mg} / \mathrm{L}$ \\
\hline Turbidity & EPA-180.1 & 0.1 & NTU \\
\hline CHEM -Anions $* \%$ \% & $\because \ldots, 8 \quad 1$ & अ & $32^{\circ}$ \\
\hline Alkalinity - $\mathrm{HCO} 3$ & $\mathrm{EPA}-310.1$ & 1.0 & $\mathrm{mg} / \mathrm{L}$ \\
\hline Alkalinity - CO3 & EPA-310.1 & 1.0 & $\mathrm{mg} / \mathrm{L}$ \\
\hline Chloride & $\mathrm{EPA}-300.0$ & 0.2 & $\mathrm{mg} / \mathrm{L}$ \\
\hline Fluoride & EPA-340.2 & 0.1 & $\mathrm{mg} / \mathrm{L}$ \\
\hline Nitrate (as Nitrogen) & EPA-300.0 & 0.028 & $\mathrm{mg} / \mathrm{L}$ \\
\hline Sulfate & EPA-300.0 & 0.25 & $\mathrm{mg} / \mathrm{L}$ \\
\hline MET(1) $=$ Metals: Unfiltered \& Filtered 4 : & ४ & औै: & स: \\
\hline Aluminum & EPA-6010A & 0.02 & $\mathrm{mg} / \mathrm{L}$ \\
\hline Antimony & EPA-200.8 & 0.005 & $\mathrm{mg} / \mathrm{L}$ \\
\hline Arsenic & EPA-200.8 & 0.005 & $\mathrm{mg} / \mathrm{L}$ \\
\hline Barium & EPA-6010A & 0.001 & $\mathrm{mg} / \mathrm{L}$ \\
\hline Beryllium & EPA-6010A & 0.0003 & $\mathrm{mg} / \mathrm{L}$ \\
\hline Boron & EPA-6010A & 0.004 & $\mathrm{mg} / \mathrm{L}$ \\
\hline Cadmium & EPA-200.8 & 0.0005 & $\mathrm{mg} / \mathrm{L}$ \\
\hline Calcium & EPA-6010A & 0.008 & $\mathrm{mg} / \mathrm{L}$ \\
\hline Chromium & EPA-6010A & 0.01 & $\mathrm{mg} / \mathrm{L}$ \\
\hline Cobalt & EPA-6010A & 0.005 & $\mathrm{mg} / \mathrm{L}$ \\
\hline Copper & EPA-6010A & 0.004 & $\mathrm{mg} / \mathrm{L}$ \\
\hline Iron & EPA-6010A & 0.005 & $\mathrm{mg} / \mathrm{L}$ \\
\hline Lead & EPA-200.8 & 0.0005 & $\mathrm{mg} / \mathrm{L}$ \\
\hline Lithium & EPA-6010A & 0.004 & $\mathrm{mg} / \mathrm{L}$ \\
\hline Magnesium & EPA-6010A & 0.003 & $\mathrm{mg} / \mathrm{L}$ \\
\hline Manganese & EPA-6010A & 0.001 & $\mathrm{mg} / \mathrm{L}$ \\
\hline Mercury & EPA-7470 & 0.0002 & $\mathrm{mg} / \mathrm{L}$ \\
\hline Molybdenum & EPA-6010A & 0.01 & $\mathrm{mg} / \mathrm{L}$ \\
\hline
\end{tabular}


Table 2 (cont'd)

\begin{tabular}{|c|c|c|c|}
\hline MET(1)-Metals (cont'd) $/ \curvearrowright$ । & Analytical Method & Detection Limit ${ }^{2}$ & Units ${ }^{3}$ \\
\hline Nickel & EPA-6010A & 0.01 & $\mathrm{mg} / \mathrm{L}$ \\
\hline Potassium & EPA-6010A & 0.6 & $\mathrm{mg} / \mathrm{L}$ \\
\hline Selenium & EPA-200.8 & 0.01 & $\mathrm{mg} / \mathrm{L}$ \\
\hline Silver & EPA-6010A & 0.006 & $\mathrm{mg} / \mathrm{L}$ \\
\hline Sodium & EPA-6010A & 0.02 & $\mathrm{mg} / \mathrm{L}$ \\
\hline Strontium & EPA-6010A & 0.0004 & $\mathrm{mg} / \mathrm{L}$ \\
\hline Thallium & EPA-200.8 & 0.0005 & $\mathrm{mg} / \mathrm{L}$ \\
\hline Thorium & EPA-6010A & 0.2 & $\mathrm{mg} / \mathrm{L}$ \\
\hline Uranium & EPA-200.8 & 0.0005 & $\mathrm{mg} / \mathrm{L}$ \\
\hline Vanadium & EPA-6010A & 0.005 & $\mathrm{mg} / \mathrm{L}$ \\
\hline Zinc & EPA-6010A & 0.002 & $\mathrm{mg} / \mathrm{L}$ \\
\hline VoC(1)-Volatile Organic Compounds & \%०: :० & $\%$ CRQL & : \\
\hline Acetone & EPA-8260 & 10 & $\mu \mathrm{g} / \mathrm{L}$ \\
\hline Acrolein & EPA-8260 & 10 & $\mu \mathrm{g} / \mathrm{L}$ \\
\hline Acrylonitrile & EPA-8260 & 10 & $\mu \mathrm{g} / \mathrm{L}$ \\
\hline Benzene & EPA-8260 & 5 & $\mu \mathrm{g} / \mathrm{L}$ \\
\hline Bromochloromethane & EPA-8260 & 10 & $\mu \mathrm{g} / \mathrm{L}$ \\
\hline Bromodichloromethane & EPA-8260 & 5 & $\mu \mathrm{g} / \mathrm{L}$ \\
\hline Bromoform & EPA-8260 & 5 & $\mu \mathrm{g} / \mathrm{L}$ \\
\hline Bromomethane & EPA-8260 & 10 & $\mu \mathrm{g} / \mathrm{L}$ \\
\hline 2-Butanone & EPA-8260 & 10 & $\mu \mathrm{g} / \mathrm{L}$ \\
\hline Carbon disulfide & EPA-8260 & 5 & $\mu \mathrm{g} / \mathrm{L}$ \\
\hline Carbon tetrachloride & EPA-8260 & 5 & $\mu \mathrm{g} / \mathrm{L}$ \\
\hline Chlorobenzene & EPA-8260 & 5 & $\mu \mathrm{g} / \mathrm{L}$ \\
\hline Chloroethane & EPA-8260 & 10 & $\mu \mathrm{g} / \mathrm{L}$ \\
\hline 2-Chloroethyl vinyl ether & EPA-8260 & 10 & $\mu \mathrm{g} / \mathrm{L}$ \\
\hline Chloroform & EPA-8260 & 5 & $\mu \mathrm{g} / \mathrm{L}$ \\
\hline Chloromethane & EPA-8260 & 10 & $\mu g / L$ \\
\hline Dibromochloromethane & EPA-8260 & 5 & $\mu \mathrm{g} / \mathrm{L}$ \\
\hline 1,2-Dibromo-3-chloropropane & EPA-8260 & 10 & $\mu \mathrm{g} / \mathrm{L}$ \\
\hline 1,2-Dibromoethane & EPA-8260 & 5 & $\mu \mathrm{g} / \mathrm{L}$ \\
\hline Dibromomethane & EPA-8260 & 10 & $\mu \mathrm{g} / \mathrm{L}$ \\
\hline 1,2-Dichlorobenzene & EPA-8260 & 5 & $\mu \mathrm{g} / \mathrm{L}$ \\
\hline 1,4-Dichlorobenzene & EPA-8260 & 5 & $\mu \mathrm{g} / \mathrm{L}$ \\
\hline 1,4-Dichloro-2-butene & EPA-8260 & 5 & $\mu \mathrm{g} / \mathrm{L}$ \\
\hline trans-1,4-Dichloro-2-butene & EPA-8260 & 5 & $\mu \mathrm{g} / \mathrm{L}$ \\
\hline Dichlorodifluoromethane & EPA-8260 & 5 & $\mu \mathrm{g} / \mathrm{L}$ \\
\hline 1,1-Dichloroethane & EPA-8260 & 5 & $\mu \mathrm{g} / \mathrm{L}$ \\
\hline 1,2-Dichloroethane & EPA-8260 & 5 & $\mu \mathrm{g} / \mathrm{L}$ \\
\hline 1,1-Dichloroethene & EPA-8260 & 5 & $\mu \mathrm{g} / \mathrm{L}$ \\
\hline 1,2-Dichloroethene & EPA-8260 & 5 & $\mu \mathrm{g} / \mathrm{L}$ \\
\hline
\end{tabular}


Table 2 (cont'd)

\begin{tabular}{|c|c|c|c|}
\hline Voc (1)-Volatile Organic Compounds (cont?d) & Analytical Method ${ }^{1}$ & CROL & Units $^{3}$ \\
\hline cis-1,2-Dichloroethene & EPA-8260 & 5 & $\mu \mathrm{g} / \mathrm{L}$ \\
\hline trans-1,2-Dichloroethene & EPA-8260 & 5 & $\mu \mathrm{g} / \mathrm{L}$ \\
\hline 1,2-Dichloropropane & EPA-8260 & 5 & $\mu \mathrm{g} / \mathrm{L}$ \\
\hline cis-1,3-Dichloropropene & EPA-8260 & 5 & $\mu \mathrm{g} / \mathrm{L}$ \\
\hline trans-1,3-Dichloropropene & EPA-8260 & 5 & $\mu \mathrm{g} / \mathrm{L}$ \\
\hline Dimethylbenzene & EPA-8260 & 5 & $\mu \mathrm{g} / \mathrm{L}$ \\
\hline Ethanol & EPA-8260 & 500 & $\mu \mathrm{g} / \mathrm{L}$ \\
\hline Ethylbenzene & EPA-8260 & 5 & $\mu \mathrm{g} / \mathrm{L}$ \\
\hline Ethyl methacrylate & EPA-8260 & 5 & $\mu \mathrm{g} / \mathrm{L}$ \\
\hline 2-Hexanone & EPA-8260 & 10 & $\mu \mathrm{g} / \mathrm{L}$ \\
\hline Iodomethane & EPA-8260 & 5 & $\mu \mathrm{g} / \mathrm{L}$ \\
\hline 4-Methyl-2-pentanone & EPA-8260 & 10 & $\mu \mathrm{g} / \mathrm{L}$ \\
\hline Methylene chloride & EPA-8260 & 5 & $\mu \mathrm{g} / \mathrm{L}$ \\
\hline Styrene & EPA-8260 & 5 & $\mu g / \mathrm{L}$ \\
\hline 1,1,1,2-Tetrachloroethane & EPA-8260 & 10 & $\mu \mathrm{g} / \mathrm{L}$ \\
\hline 1,1,2,2-Tetrachloroethane & EPA-8260 & 5 & $\mu \mathrm{g} / \mathrm{L}$ \\
\hline Tetrachloroethene & EPA-8260 & 5 & $\mu g / L$ \\
\hline Toluene & EPA-8260 & 5 & $\mu \mathrm{g} / \mathrm{L}$ \\
\hline 1,1,1-Trichloroethane & EPA-8260 & 5 & $\mu \mathrm{g} / \mathrm{L}$ \\
\hline 1,1,2-Trichloroethane & EPA-8260 & 5 & $\mu \mathrm{g} / \mathrm{L}$ \\
\hline Trichloroethene & EPA-8260 & 5 & $\mu g / L$ \\
\hline Trichlorofluoromethane & EPA-8260 & 5 & $\mu g / L$ \\
\hline 1,2,3-Trichloropropane & EPA-8260 & 5 & $\mu \mathrm{g} / \mathrm{L}$ \\
\hline Vinyl acetate & EPA-8260 & 10 & $\mu \mathrm{g} / \mathrm{L}$ \\
\hline Vinyl chloride & EPA-8260 & 10 & $\mu \mathrm{g} / \mathrm{L}$ \\
\hline Radiological Analytes (pCi/L) & \% \% & Target MDA & िएथ \\
\hline Gross Alpha Activity & EPA-900.0 & 3.5 & $\mathrm{pCi} / \mathrm{L}$ \\
\hline Gross Beta Activity & EPA-900.0 & 7.0 & $\mathrm{pCi} / \mathrm{L}$ \\
\hline Strontium-89/90 & EPA-905.0 & 4.0 & $\mathrm{pCi} / \mathrm{L}$ \\
\hline Technetium-99 & Y/P65-7154 & 10 & $\mathrm{pCi} / \mathrm{L}$ \\
\hline $\operatorname{RAD(2)}$ & EPA-906.0 & 300 & $\mathrm{pCi} / \mathrm{L}$ \\
\hline Uranium-234, 235, \& 238 & AC-MM-2-22012 & 0.4 & $\mathrm{pCi} / \mathrm{L}$ \\
\hline Americium-241 & AC-MM-2-22012 & 0.4 & $\mathrm{pCi} / \mathrm{L}$ \\
\hline Iodine-129 & EPA-901.1 & 3.0 & $\mathrm{pCi} / \mathrm{L}$ \\
\hline Neptunium-237 & Y/P65-7206 & 0.4 & $\mathrm{pCi} / \mathrm{L}$ \\
\hline Plutonium-238 \& 239/240 & AC-MM-2-22012 & 0.4 & $\mathrm{pCi} / \mathrm{L}$ \\
\hline Radium-223/224/226 & EPA-903.0 - 904.0 & 0.5 & $\mathrm{pCi} / \mathrm{L}$ \\
\hline Thorium-228,230,232, \& 234 & Y/P65-7206 & 0.4 & $\mathrm{pCi} / \mathrm{L}$ \\
\hline Total Uranium and weight $\%$ U-235 & Y/P65-8044 & NA & $\mathrm{mg} / \mathrm{L}$ \\
\hline
\end{tabular}




\section{Table 2 (cont'd)}

\section{Notes:}

1 Analytical/field methods/procedures from:

- Test Methods for Evaluating Solid Waste Physical/Chemical Methods (U.S. Environmental Protection Agency 1986)

- Methods for Chemical Analysis of Water and Wastes (U.S. Environmental Protection Agency 1983)

- Environmental Surveillance Procedures Quality Control Manual (Lockheed Martin Energy Systems, Inc. 1988)

- Lockheed Martin Energy Systems ASO radiological methods

2 NA - not applicable

$3 \mathrm{ft}$ - feet

$\mu \mathrm{g} / \mathrm{L}$ - micrograms per liter

$\mu \mathrm{mho} / \mathrm{cm}$ - micromhos per centimeter

$\mathrm{mg} / \mathrm{L}$ - milligrams per liter

$\mathrm{mV}$ - millivolts

NTU - nephelometric turbidity units

ppm - parts per million

$\mathrm{pCi} / \mathrm{L}$ - picoCuries per liter

$4 \quad$ Filtered samples will be collected only at specified locations (see Table 1). Groundwater samples for dissolved metals analysis collected with dedicated sampling equipment or portable pumps will be filtered in the field; samples collected with bailers and surface water samples will be filtered in the laboratory.

$5 \mathrm{CRQL}$ - contract-required quantitation limit

$6 \mathrm{MDA}$ - minimum detectable activity. The target MDA may be obtained under optimal analytical conditions; actual MDAs are sample-specific and may vary significantly from the target value. 